\section{REFERENCES}

BITTERMAN M. E. Phyletic differences in leaming. American Psychologist, 1965. 20, 396-410.

DOOLITTLE. J. H. \& WEIMER, J. Spatial probability leaming in the Virginia opossum. Psychonomic Science, 1968. 13, 191

FRIEDMAN, H., \& MARSHALL, D. A. Position reversal training in the Virginia opossum: Evidence for the acquisition of a learning set. Quarterly Joumal of Experimental Psychology, 1965, 17 $250-254$.

HAMILTON,W. J., JR. Success story of the opossum. Natural History, 1963, 72 , 16-25

HERRING, F, H. MASON, D. J.,
DOOLITTLE. J. H., \& STARRETT, D, E The Virginja opossum in psychological research Psychological Reports, 1966 , 19. 755-757.

SIMPSON, G. G. The beginning of the age of mammals. Biological Reviews, 1937. $12,1.47$.

VORONIN, L. G. Some results of comparative-physiological investigations of higher nervous activity. Psychological Bulletin, 1962, 59, 161-195.

WARREN, J. M . The comparative psychology of leaming. Annual Review of Psychology, 1965, 16,95-118.

WICKENS, D. D., \& MILES, R. C Extinction changes during a series of reinforcement-extinction sessions. Journal of Comparative \& Physiologica Psychology, 1954,47, 315-317.

\title{
Food-satiation response functions of albino rats, obtained on a three-valued randomized deprivation schedule
}

\author{
ROBERT L. GOSSETTE \\ Comparative Animal Behavior Laboratory \\ Hofstra University, Hempstead, N.Y. 11550
}

Food-satiation response functions of albino rats, obtained under a three-valued randomized food-deprivation schedule, were analyzed. The method of analysis focused upon transforming response and time measures to a common scale. This revealed a considerable regularity across satiation values. Rate declines typical of satiation would appear to reflect a lengthening of feeding pauses that are interspersed among bursts of feeding responses.

The study of satiation functions, 1 notably those involving food or water consummatory responses, has been of perennial interest for many years. Analysis of such functions is vital not only for the information they provide about the variables controlling feeding and drinking behavior, but also for a more adequate appraisal of the methodological appropriateness of dependent variables that might be employed in the experimental scrutiny of a number of different environmental and organismic variables. For example, one of the most widely used measures is response rate in free operant tasks. If the reinforcement schedule employed approaches continuous reinforcement, variation in response rate may reflect properties of satiation as much as the experimental variables explicitly selected for study. Systematic inflections in rate mistakenly attributed to the experimental variable may in fact represent characteristics of the satiation function. On the other hand, in the comparative analysis of behavior, obtained interspecies performance differences emerging from methodology utilizing consummatory responses may not reflect differences in, for example, learning ability, threshold sensitivity, or even species-specific repertoires, but rather interspecies variation in properties of satiation. Furthermore, through detailed interspecies comparisons of satiation functions, it may be possible to devise a calibration procedure by which different species may be equated along motivational-incentive variables. Accordingly, analyses of satiation, both across and within species, are of considerable interest. As an initial exploration of the usefulness of the satiation in comparative analyses, a detailed description of satiation behavior of rats was undertaken.

\section{SUBJECTS}

The Ss were six albino rats, two female and four males, about 130 days old. Ss were confined in the test chambers throughout the experiment and removed only for daily weighing. APPARATUS

Apparatus consisted of a single bank of operant chambers located in a well-illuminated windowless room. Each chamber was 8 in. wide, 10 in. long, and 6 in. deep. $A$ bar manipulandum rested in grooved supports attached to the chamber front to permit ready insertion and withdrawal. Two chambers were employed concurrently, with reinforcement consisting of $45-\mathrm{g}$ Noyes pellets released by a Gerbrand pellet dispenser. Water was continuously available. Barpressing was recorded on a four-channel event recorder. One channel received a 15 -sec time pulse to permit temporal calibration of barpressing.

Three values of food deprivation were chosen: 2, 11, and $23 \mathrm{~h}$. Deprivation was measured from the last reinforced response of the last satiation to the initiation of the next session. Each $S$ was assigned a randomized schedule containing the three values of deprivation. Following a 6-day habituation period of ad lib feeding, each $S$ was placed on deprivation and trained to barpress for food. Once barpressing was established, food was obtained only through barpressing. Satiation measurement continued for about $2 \frac{1}{2}$ months. A S was considered satiated when an interval of 9 min had elapsed without a response.

\section{RESULTS AND DISCUSSION}

Conventional analysis of satiation involving total time (T) and responses (R) to satiate and initial rate (IR) of satiation has generally reported that all of these measures are a negatively accelerated increasing function of food deprivation. The data of this experiment are no exception-Table 1 . The focus of the present analysis, however, is upon parameters of the

Table 1

Mean Total Responses (R) and Total Minutes ( $T$ ) and the Initial Rate (IR) Across Three Values of Food Deprivation for Six Ss. Each mean is based on 17 sessions for each $S$.

\begin{tabular}{rlrrr}
\hline \multirow{2}{*}{$\begin{array}{r}\text { Response } \\
\text { Measures }\end{array}$} & \multicolumn{3}{c}{ Hours of Food Deprivation } \\
\cline { 3 - 5 } R & M & $\mathbf{2}$ & \multicolumn{1}{c}{11} & \multicolumn{1}{c}{23} \\
\hline \multirow{2}{*}{ T } & SD & 50.3 & 228.6 & 312.5 \\
& M & 18.1 & 78.2 & 55.8 \\
& SD & 10.5 & 15.4 & 12.0 \\
\multirow{2}{*}{ IR } & M & 18.4 & 27.0 & 29.9 \\
& SD & 8.8 & 8.4 & 7.0 \\
\hline
\end{tabular}




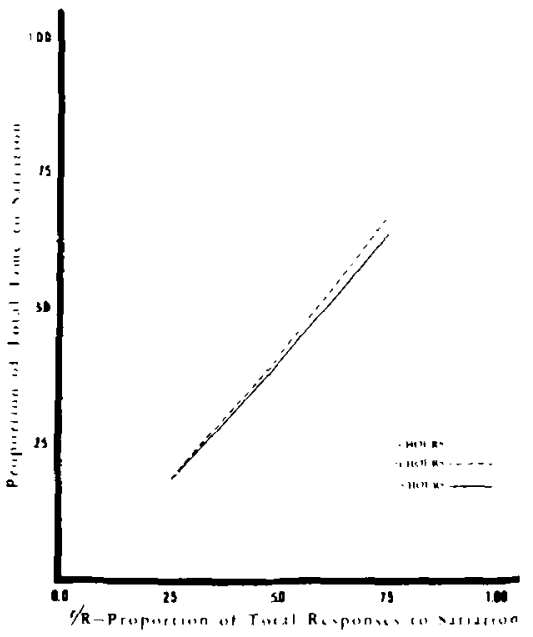

Fig. 1. Mean $t / T$ values for $.25, .50$, and .75 of $R$ across three levels of food deprivation. See text for expla. nation of variables.

satiation function itself. To reduce the "noise" contributed by both intra-S and inter-S variability in $R$ and $T$ values, the record of each satiation session was transformed as follows: Both response and time values within the satiation session were transformed into proportions of the particular $R$ and $T$ scores for that session. That is, the cumulative value for each successive 3-min interval was computed for both measures and divided by the appropriate $R$ and $T$ scores for that session. This resulted in intrasession scales, which specified for each successive 3 -min interval the proportion of total responses $(r / R)$ already made and the proportion of the total satiation time (t/T) that had already elapsed.

Three reference values of $r / R$ were selected for analysis: $.25, .50$, and .75 , representing, respectively, one-quarter, one-half, and three-quarters of the S's total satiation function, or food intake. The last five satiation sessions of each $S$ at each level of deprivation were selected for the analysis as follows: The specific $t / T$ values corresponding to $r / R$ values of .25 , .50 , and .75 for each session were computed. The mean $\mathrm{t} / \mathrm{T}$ values for these three points along the satiation function, across deprivation levels, are shown in Fig. 1. As can be seen, they exhibit considerable linearity. However, it should be noted that this linearity must, of necessity, be restricted to the middle sector of the satiation function, with an acceleration along the inital sector, e.g., from $r / R=.00$ to $r / R=.25$, and a deceleration along the terminal sector of the function, e.g., $r / R$ values from .75 to 1.00 . Examination of $r / R$ value of .50 , which we will call the "half-feed" intake, reveals that only at $2 \cdot h$ deprivation does it approach the "half-feed" time, $t / T$ value of .50 . With higher deprivation values, it moves toward the anterior portion of the function. The same is true for oneand three-quarters feed intakes.

The nature of the curvilinearity of the ends of the function is more directly illustrated by Fig. 2, which shows rate of response, measured over 3-min intervals along the satiation function from $r / R$ values, 00 to 1.00 . The data shown are from one $S$, taken from the last five sessions at each level of deprivation. As can be seen, response rate is elevated over the anterior, and depressed over the terminal sectors of the function, especially for the 11 - and 23-h deprivations. Figure 2 also suggests that the linearity noted above is actually that of a slight, but constant, decline in response rate. As a more direct test, rates at the three values of $r / R$ were computed for all Ss over the last five sessions at each level of deprivation. Analysis of variance yielded significance $(F=5.75$, $p<.01)$ only for the deprivation variable, but as can be seen from Fig. 2B, there is a tendency for midsatiation rates to undergo a slight linear decline.

Thus, the satiation function displays considerable regularity, despite wide intra- and inter-S variability, when

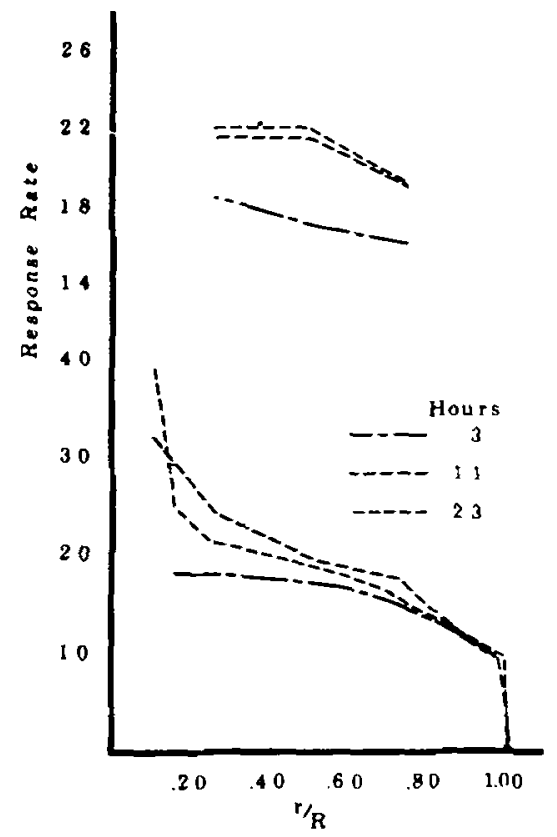

Fig. 2. (A) Mean rate of satiation, measured at three values of $r / R, .25$, .50 , and .75 , at three levels of deprivation. Each point is the mean of 30 scores. (B) Mean rate of satiation for one $S$ at three levels of deprivation plotted over $r / R$. Rate measured over 3-min intervals. transformed as described above. Typically, albino rats will eat one-half of their ration within about $40 \%$ of the total time that will be required to satiate. This "half-feed" time appears to be quite stable, showing but a slight lengthening at low deprivation levels, e.g., $2 \mathrm{~h}$.

About the same value has been found with birds. ${ }^{2}$ While response rate has been shown above to undergo change from a high initial value through a slight linear midsatiation decline to a rather rapid termmal deceleration, a rather informal examination of interresponse times suggests that these rate changes reflect the interaction of two more basic processes, each of which is under the control of different variables. A distinction can be made between a feeding burst and a feeding pause. A feeding burst consists of a series of responses with characteristically short interresponse times. A feeding pause is a separation between successive feeding bursts, e.g., an interresponse time that exceeds that typical of a burst. Interestingly, the intraburst interresponse times are strikingly similar across all sectors of the satiation fusction. The feeding pause, however, appears to gradually lengthen as intake increases, with duration of the feeding burst progressively shortening. A similar observation has been reported by Sidman \& Stebbins (1954). A more detailed and rigorous study of this phenomenon is now under way.

One practical, and methodological, consequence of this form of analysis of satiation would appear to be the questioning of the adequacy of response rate as a dependent variable when obtained under reinforcement schedules that lead to satiation, for under these conditions, rates may more readily reflect parameters of satiation, e.g., feeding bursts, etc., and not the experimental variables being studied. A similar conclusion has been reported for drinking responses (Stellar \& Hill, 1952).

\section{REFERENCES}

SIDMAN, M.. \& STEBQINS, W. C. Satiation effects under fixed-ratio schedules of reinforcement. Journal of Comparative Physiological Psychology, 1954, 47. $114-116$.

STELLAR, E., \& HILL, J. H. The ratio rate of drinking as a function of water deprivation. Journal of Comparative \& Physiological Psychology. 1952, 45, 96-102.

\section{NOTES}

1. The term satiation function will refer to any continuous variation in response measures following a period of deprivation from the initiation to the cessation of eating as defined by satiation criterion, c.g., $9 \mathrm{~min}$ of no further responding.

2. Gossette, R. Similarity of satiation functions across different species of birds. Submitted for publication. 